
\title{
25 Research Square \\ Comparison Efficacy of Bacterial and Fungal Probiotics on Acute Pediatric Diarrhea A Randomized Clinical Trial
}

\author{
Alireza Sharif \\ Kashan University of Medical Sciences \\ Abbas Taghavi Ardakani \\ Kashan University of Medical Sciences \\ Hamed Haddad Kashani \\ Kashan University of Medical Sciences \\ Fatemeh Rabiee \\ Kashan University of Medical Sciences \\ Mohammad Reza Sharif ( $\square$ sharif.kaumsc@gmail.com ) \\ Kashan University of Medical Sciences
}

Research article

Keywords: Diarrhea; Probiotic; Fungi; Bacteria; Children; Health.

Posted Date: July 16th, 2019

DOI: https://doi.org/10.21203/rs.2.11286/v1

License: (9) This work is licensed under a Creative Commons Attribution 4.0 International License. Read Full License 


\section{Abstract}

Background Diarrheal disease is one of the leading causes of mortality and morbidity in developing countries. Probiotics, recognized as living microorganisms, are known to balance human intestinal flora. Many studies have shown that probiotics can be used as an alternative treatment to acute diarrhea. This study was designed to evaluate the effects of fungal and bacterial probiotics on acute pediatric diarrhea. Methods This clinical trial was conducted on 90 children aged 2 to 5 years, who were referred to the Kashan Children's Hospital, Iran, in 2015 for complaints of acute watery diarrhea and were treated on an outpatient basis. These children were randomly divided into three groups of 30 . The first group received a standard daily treatment with ORS plus bacterial probiotics as a Kidilact ${ }^{\circledR}$ sachet containing 7 strains of Lactobacillus strains; the second group received ORS and fungal probiotics containing Saccharomyces boulardii as Ardeypharm Yomogi® capsule, $250 \mathrm{mg}$ once a day and the third group used placebo along with standard oral rehydration therapy. The duration of treatment with probiotics or placebo was 5 days. Results The current study showed no significant differences in age, gender, frequency and duration of diarrhea between the three groups before admission. The mean duration of diarrhea from onset of treatment to recovery was 2.80 days in the first group (bacterial probiotics), 3.17 days in the second group (fungal probiotics), and 4.43 days in the third group (placebo). A significant difference was observed in the duration of diarrhea after treatment between the three groups $(P \leq 0.05)$, so that the best effect was found in reducing the duration of diarrhea in the bacterial probiotic group, and the lowest effect was associated with the placebo group. Conclusion The use of probiotics in acute diarrhea in children could be effective in shortening the duration of diarrhea; however, the results of this study indicate that bacterial probiotics may seems to be superior to fungi but statistically does not have significant differences.

\section{Abstract}

Background: Diarrheal disease is one of the leading causes of mortality and morbidity in developing countries. Probiotics, recognized as living microorganisms, are known to balance human intestinal flora. Many studies have shown that probiotics can be used as an alternative treatment to acute diarrhea. This study was designed to evaluate the effects of fungal and bacterial probiotics on acute pediatric diarrhea.

Methods: This clinical trial was conducted on 90 children aged 2 to 5 years, who were referred to the Kashan Children's Hospital, Iran, in 2015 for complaints of acute watery diarrhea and were treated on an outpatient basis. These children were randomly divided into three groups of 30 . The first group received a standard daily treatment with ORS plus bacterial probiotics as a Kidilact ${ }^{\circledR}$ sachet containing 7 strains of Lactobacillus strains; the second group received ORS and fungal probiotics containing Saccharomyces boulardii as Ardeypharm Yomogi ${ }^{\circledR}$ capsule, $250 \mathrm{mg}$ once a day and the third group used placebo along with standard oral rehydration therapy. The duration of treatment with probiotics or placebo was 5 days.

Results: The current study showed no significant differences in age, gender, frequency and duration of diarrhea between the three groups before admission. The mean duration of diarrhea from onset of 
treatment to recovery was 2.80 days in the first group (bacterial probiotics), 3.17 days in the second group (fungal probiotics), and 4.43 days in the third group (placebo). A significant difference was observed in the duration of diarrhea after treatment between the three groups $(P \leq 0.05)$, so that the best effect was found in reducing the duration of diarrhea in the bacterial probiotic group, and the lowest effect was associated with the placebo group.

Conclusion: The use of probiotics in acute diarrhea in children could be effective in shortening the duration of diarrhea; however, the results of this study indicate that bacterial probiotics may seems to be superior to fungi but statistically does not have significant differences.

Clinical trial registration: IRCT2013031511145N6. Registered on August 31, 2013.

Keywords: Diarrhea; Probiotic; Fungi; Bacteria; Children; Health.

\section{Background}

Diarrheal disease is the leading cause of death in developing countries, killing more than 3 million children each year. According to the classification list used by the Department of Health, diarrhea was registered as the second leading cause of child mortality and morbidity. The World Health Organization (WHO) defined diarrhea as the frequent passage of watery, loose stools or more than three times daily fluid bowel movements, with the change in stool consistency being considered more important than the frequency of defecation, especially in the first few months of life, compared to the previous form [1-3].

Essential factors recommended by the WHO for the control of acute gastroenteritis include the use of ORS with reduced osmolarity, intravenous fluids and, optionally, zinc supplements that may decrease the duration of treatment and the severity of diarrhea. Administration of ORS may reduce mortality and morbidity due to diarrhea, but does not affect the duration of treatment and the severity of the disease [13].

Many studies have shown that the use of probiotics is an alternative treatment for diarrhea. The term "probiotics" comes from the Greek and means "life". The term was first used by Lyle and Steelville in 1965 to describe the enhancement of one microorganism's growth by another. Later, various definitions of probiotics were proposed up to Fuller's new definition of probiotics in 1985: "Active microbial feed additives have a beneficial effect on host animals by improving their microbial gut balance." WHO introduces probiotics as "living microorganisms" which, when administered in sufficient quantities, confer health benefits to the host [4-8].

Nowadays, probiotics are used in a variety of fields, especially in the prevention and treatment of acute infectious diarrhea. The results of a systematic review of 56 studies have shown that the use of probiotics in infants and children is safe and significantly reduces the duration and frequency of acute infectious diarrhea [9]. 
The mechanism and effectiveness of probiotics usually depend on the interaction of the probiotic microorganisms with the individual's flora and immune cells of the intestinal mucosa. The best evidence of probiotics has been discussed in several previous clinical trials by stimulating the immune system to eliminate the duration of symptoms and acute infections. The role of probiotics includes stimulation of the immune system, reduction of competition for intestinal nutrition, adhesion to intestines and mucous membranes, prevention of harmful substances, production of antibacterial substances $\left(\mathrm{H}_{2} \mathrm{~S}\right.$, bacitracin and fatty acids) and the elimination of harmful microbial digestive systems by deconjugating of bile salts and $\mathrm{pH}$ reduction [10-12].

This study was designed to assess the effects of available probiotics on acute pediatric diarrhea in Kashan, Iran. A large number of hospital beds are dedicated annually in Kashan, as are many parts of Iran and other countries to acute gastroenteritis, and is the main complaint among outpatient referrals to children's hospitals. Given that no similar studies have been conducted in this area, it is hoped that the use of probiotics will reduce the prevalence and complications of acute childhood diarrhea and the socioeconomic burden to a certain extent, as the duration of treatment will be reduced (to reduce the duration of infection) is necessary to control this disease.

\section{Methods}

The present study, registered in the Iranian website for registration of clinical trials (http://www.irct.ir: IRCT2013031511145N6). The procedures performed in the study were in accordance with the ethical standards of Academic Research Committee of Kashan University of Medical Sciences. This randomized clinical trial conducted on 90 children between the ages of 2 and 5, who were referred to the Kashan Children's Hospital in the year of 2015 for complaints of acute watery diarrhea and were treated on an outpatient basis. The inclusion criteria were patients with loose, watery and non-bleeding stools, often more than three times daily and between two and five years of age.

Subjects were assigned to three groups of 30 whose parents signed the consent form to participate in the study. First, follow-up and systemic examinations were performed on the children and the required information was recorded. Patients were compared to the frequency of bowel movements 24 hours before the start of the study.

The first group received a standard treatment with ORS plus bacterial probiotics as Kidilact ${ }^{\circledR}$ sachet containing 7 strains of Lactobacillus species with concentration of $10^{9} \mathrm{CFU}$ (Colony Forming Unit) and including of Lactobacillus casei, Lactobacillus acidophilus, Lactobacillus rhamnosus, Lactobacillus bulgaricus, Bifidobacterium infantis, Bifidobacterium breve, Streptococcus thermophiles and a prebiotic product of fructooligosaccharides (FOS) consumed once a day.

The second group received ORS and fungal probiotics once daily containing Saccharomyces boulardiias Ardeypharm Yomogi $i^{\circledR}$ Capsule $250 \mathrm{mg}$ at a concentration of $2.5 \times 10^{9} \mathrm{CFU}$ and the third group a placebo with a standard oral rehydration therapy. 
The placebo matched the taste and color with the probiotics, but with no positive or negative effects on the patients. Each of these medications (bacterial probiotics, fungal prebiotics and placebo) was given to the mothers for five days. The study was a single-blind study in which mothers were unaware of the nature of the drugs. Criteria for considering the patients as cured were a reduction in the frequency of bowel movements up to three times a day or normal stool consistency compared to before diarrhea. Finally, the mean duration of diarrhea from start of treatment to full recovery in each of the groups was compared to the control group.

\section{Statistical analysis}

After collecting the required data, the results were analyzed with SPSS 24 software. The KolmogorovSmirnov test was used to evaluate the normality of the data. All data in this study was normal. The ANOVA test was recruited to determine the statistical difference between the three groups (the first group (bacterial probiotics), the second group (fungal probiotics), and the third group (placebo).

In addition, the present study used a two-sample independent t-test to compare the mean duration of diarrhea from the beginning of treatment to the recovery between bacterial and fungal groups, and the Duncan test was also used to compare the minimum and the maximum diarrhea duration in three groups. Based on mean and statistics, the respective three groups were divided by Duncan's test into two homogenous bacterial and fungal probiotics groups and the placebo group. In other words, the Duncan test showed that the average duration of diarrhea between the placebo group and the bacterial and fungal probiotic groups was significantly different from the onset of treatment to recovery. In the present study, the P-value with a confidence interval of $95 \%$ was considered to be $0.05(P \leq 0.05)$.

Figure 1.

\section{Results}

In the current study, the mean duration of diarrhea before admission was 6.28 (SD = 1.722), the mean frequency of diarrhea before 24 hours was 1.91 (SD = 0.802), and the mean duration of diarrhea from the start of treatment to recovery was $3.47(S D=1.813)$. The average age of the child is 31.1 months. Of the 90 patients enrolled, 49 (54.4\%) were male and 41 (45.6\%) were female.

The results showed that the mean age of the first group (bacterial probiotics) was 30.87 months, the second group (fungal probiotics) was 30.37 months, and the third group (placebo) was 32.07 months. The mean diarrhea rate before 24 hours was 1.73 days in the first group (bacterial probiotic), 2.10 days in the second group (fungal probiotic) and 1.90 days in the third group (placebo). In addition, the median duration of diarrhea from onset of treatment to recovery was 2.80 days in the first group (bacterial probiotic), 3.17 days in the second group (fungal probiotic), and 4.43 days in the third group (placebo). The mean duration of diarrhea before admission was 6.63 days in the first group (bacterial probiotic), 6.03 days in the second group (fungal probiotic), and 6.17 days in the third group (placebo). 
In order to compare gender among the groups, table 1 (in the Supplementary Files) presents the results of one-way ANOVA test regarding the gender differences among the three statistical groups. As can be seen, the significant level of Fisher's test value $(F=0.57)$ is higher than the obtained error rate of 0.05 . Hence, it can be stated that gender of the three statistical groups was not significantly different.

Table 2 (in the Supplementary Files) shows that the duration of diarrhea prior to admission has no statistically significant difference in the three groups.

As displays in table 3 (in the Supplementary Files) the frequency of diarrhea 24 hours prior to admission in three statistical groups had not any statistically significant difference among the three groups.

According to Table 3, it was found that the frequency of diarrhea in the 24 hours before admission was statistically identical in all three groups.

Table 4 (in the Supplementary Files) compares diarrhea duration from initiation of treatment to recovery in the three groups and shows that the significance level of the Fisher test value $(F=7.719)$ is greater than the error rate of $0.05(p=0.001)<0.05$., Therefore, it can be stated that the duration of diarrhea is not equal and has a statistically significant difference between the three groups.

Duncan's post hoc test was used to determine the groups with highest and lowest duration of diarrhea from the onset of treatment until the recovery, which the results are given in Table 5 (in the Supplementary Files).

Accordingly, Duncan's test divided the three existing groups into two homogenous groups in terms of mean values and statistical data that the first group includes the bacterial and fungal probiotics and the second group involved the placebo. In other words, Duncan's test suggests that there is a significant difference in mean diarrhea duration from the onset of treatment to recovery between the placebo group and the bacterial and fungal probiotics groups. The results in Table 5 also show that, based on the collected data and statistics, the mean duration of recovery from the onset of treatment until recovery between the two groups of bacterial probiotics and fungal probiotics was similar, without any significant difference.

Figure 2.

The results of the ANOVA test showed that the duration of diarrhea due to the treatment was not statistically equal in all three groups. Figure 2 shows differences in the duration of diarrhea from the onset of treatment until the recovery between the three groups, suggesting that the duration is lower in the bacterial group and higher in the placebo group than in the other two groups.

Table 6 (in the Supplementary Files) present the results of the independent t-test to compare the duration of diarrhea from the onset of treatment to recovery between the two bacterial and fungal groups. 
Accordingly, the significance level of the independent t-test is 0.389 , which is greater than the error rate of 0.05 , suggesting that the duration of diarrhea is same from the beginning of the treatment to recovery between the bacterial and fungal groups and has no significant difference.

The mean duration of diarrhea from the start of treatment to recovery was 3.17 days in the second group (fungal probiotics) and 2.80 days in the first group (bacterial probiotics).

\section{Discussion}

To our knowledge, the present study was the first in the Middle East to compare two different types of fungal and bacterial probiotics in acute watery diarrhea in children. Both bacterial and fungal probiotics help significantly in the treatment of pediatric diarrhea, which could lead to better patient improvement. In this study, the mean duration of diarrhea from onset of treatment to recovery was 2.80 days in the bacterial probiotic group, 3.17 days in the probiotic fungal group, and 4.43 days in the placebo group, which significant difference in duration of diarrhea after treatment was observed in three studied groups.

A study of children hospitalized for acute diarrhea at the age of 1-23 months showed that the duration of diarrhea in children who received Saccharomyces was significantly reduced. This probiotic reduced disease duration to $31.4 \%$ and fever duration to $73 \%$ [13]. Another study, which also supports our research, examined the effects of probiotics in 571 children aged 3 to 36 months with acute diarrhea. The average disease duration of children receiving probiotics was 78.5 hours, which was shorter than the control group (115 hours) receiving only ORS[1]. Francavilla study also evaluate the effect of Lactobacillus reuteri versus placebo in children with age of 6-36 months with acute diarrhea, 35 children received probiotic (L. reuteri) and 34 patients received placebo.

The diarrhea duration was 2.1 days in the probiotic group and 3.3 days in the control group. In the probiotic group, $55 \%$ and $45 \%$ of patients had diarrhea on the 2 nd and 3rd day of treatment, respectively, compared to $82 \%$ and $74 \%$ in the control group, respectively, with the exception of the length of hospital stay between two groups, the differences were statistically significant in this study [14].

In another study by Erdoğan, 75 children aged 5 to 50 months with rotavirus gastroenteritis, the first group received fungal probiotics (Saccharomyces boulardii), the second group received bacterial probiotics (Bifidobacterium lactis) and the third group received a diet without probiotics. The duration of the first group of diarrhea was 6.6 days, that of the second group 4.1 days and 7 days in the third group, indicating that the use of probiotics can significantly shorten the duration of diarrhea. In this study, the effect of $B$. lactis as a probiotic bacterium was more than fungal probiotic [15]. This results supports our finding on the effectiveness of bacterial and fungal probiotics in reducing the duration of diarrhea as well as the better effect of bacterial probiotics than fungal probiotics.

Current research was conducted on children underwent outpatient treatment, and was not affected by severe diseases. However, also in the study of Francavilla, the bacterial probiotic was effectively reduce 
the duration of diarrhea and there was no significant differences between the respective group and the placebo [14].

In the present study, no complications were observed after the use of probiotics in children and this result was highlighted in the other studies $[9,16]$. However, most studies consider the effect of Lactobacillus and Saccharomyces to be useful in the treatment of acute diarrhea [16-19], but there are needs to conduct further studies to evaluate recommending the routine consumption of probiotics for treatment of diarrhea [20].

\section{Conclusion}

The results of the present study and other studies demonstrate that the use of probiotics in diarrheal disease in children can be effective for shortening the duration of diarrhea. Based on the collected data and statistics analysis, the mean duration of diarrhea from the onset of treatment until the recovery between the two groups of bacterial probiotic and fungal probiotic is mostly similar, without any significant difference. Moreover, it seems that the bacterial probiotics was better than the fungal probiotics but statistically does not have significant differences. However, it is recommended to conduct further studies to investigate the effects and complications of bacterial and fungal probiotics in acute pediatric diarrhea, so by confirming the obtained results, conventional consumption of probiotics can be recommended for the treatment of diarrhea.

However, it is recommended to conduct further studies to investigate the combination therapy of both bacterial and fungal probiotics in acute pediatric diarrhea. The combination therapy could have a synergistic or antagonistic effect on the treatment of diarrhea.

\section{Abbreviations}

ORS: Oral Rehydration Solution

WHO: World Health Organization

CFU: Colony Forming Unit

FOS: Fructooligosaccharides

\section{Declarations}

\section{Ethics approval and consent to participate}

All procedures performed in the study involving human participants were in accordance with the ethical standards of the Academic Research Committee (ARC) of Kashan University of Medical Sciences and with the 1964 Helsinki declaration and its later amendments. This clinical research trial is part of the 
study that has been registered at www.irct.ir as (IRCT2013031511145N6). We have also obtained consent to publish from the participant (or legal parent or guardian for children) to report individual patient data. The parents were reassured that non-participation would not affect the services they received and that their child's information would be kept strictly confidential.

\section{Consent for publication}

Not applicable Consent for publication

\section{Availability of data and materials}

The dataset used in this study is available with the authors and can be made available upon request.

\section{Competing interests}

The authors declared that they have no competing interests.

\section{Funding}

The financial support for providing the medicines and probiotics in the current research was provided by Research Deputy of Kashan University of Medical Sciences, Kashan, Iran.

\section{Authors' contributions}

All the authors participated in the study design. MRS and ARS collected and documented the data and assisted in preliminary data analysis. HHK, FR, and ATA wrote the initial draft. HHK, ATA and MRS participated in draft revision, data analysis and editing of the final draft.

\section{Acknowledgments}

The researcher wishes to express his appreciation to the full cooperation of the patients and personnel who participated in this study and the Clinical Research Center of Shahid Beheshti Hospital.

\section{References}

1. Canani, R.B., et al., Probiotics for treatment of acute diarrhoea in children: randomised clinical trial of five different preparations. Bmj, 2007. 335(7615): p. 340. 
2. Ozuah, P.O., J.R. Avner, and R.E. Stein, Oral rehydration, emergency physicians, and practice parameters: a national survey. Pediatrics, 2002. 109(2): p. 259-261.

3. Cucchiara, S., et al., New therapeutic approach in the management of intestinal disease: probiotics in intestinal disease in paediatric age. Digestive and Liver Disease, 2002. 34: p. S44-S47.

4. Boirivant, M. and W. Strober, The mechanism of action of probiotics. Current opinion in gastroenterology, 2007. 23(6): p. 679-692.

5. Billoo, A., et al., Role of a probiotic (Saccharomyces boulardii) in management and prevention of diarrhoea. World journal of gastroenterology: WJG, 2006. 12(28): p. 4557.

6. Petrof, E.O., Probiotics and gastrointestinal disease: clinical evidence and basic science. AntiInflammatory \& Anti-Allergy Agents in Medicinal Chemistry (Formerly Current Medicinal Chemistry-AntiInflammatory and Anti-Allergy Agents), 2009. 8(3): p. 260-269.

7. Isolauri, E., Probiotics for infectious diarrhoea. Gut, 2003. 52(3): p. 436-437.

8. Kashani, H.H., et al., Recombinant endolysins as potential therapeutics against antibiotic-resistant Staphylococcus aureus: current status of research and novel delivery strategies. Clinical microbiology reviews, 2018. 31(1): p. e00071-17.

9. Allen, S.J., et al., Probiotics for treating acute infectious diarrhoea. Sao Paulo Medical Journal, 2011. 129(3): p. 185-185.

10. Surawicz, C.M., Probiotics, antibiotic-associated diarrhoea and Clostridium difficile diarrhoea in humans. Best Practice \& Research Clinical Gastroenterology, 2003. 17(5): p. 775-783.

11. Htwe, K., et al., Effect of Saccharomyces boulardii in the treatment of acute watery diarrhea in Myanmar children: a randomized controlled study. The American journal of tropical medicine and hygiene, 2008. 78(2): p. 214-216.

12. Dahan, S., et al., Saccharomyces boulardii interferes with enterohemorrhagic Escherichia coli-induced signaling pathways in T84 cells. Infection and immunity, 2003. 71(2): p. 766-773.

13. Grandy, G., et al., Probiotics in the treatment of acute rotavirus diarrhoea. A randomized, double-blind, controlled trial using two different probiotic preparations in Bolivian children. BMC infectious diseases, 2010. 10(1): p. 253.

14. Francavilla, R., et al., Randomised clinical trial: Lactobacillus reuteri DSM 17938 vs. placebo in children with acute diarrhoea-a double-blind study. Alimentary pharmacology \& therapeutics, 2012. 36(4): p. 363-369. 
15. Erdoğan, Ö., et al., The comparition of the efficacy of two different probiotics in rotavirus gastroenteritis in children. Journal of tropical medicine, 2012. 2012.

16. Goldenberg, J.Z., et al., Probiotics for the prevention of Clostridium difficile-associated diarrhea in adults and children. The Cochrane Library, 2013.

17. Applegate, J.A., et al., Systematic review of probiotics for the treatment of community-acquired acute diarrhea in children. BMC public health, 2013. 13(3): p. S16.

18. Bernaola Aponte, G., et al., Probiotics for treating persistent diarrhoea in children. Cochrane Database Syst Rev, 2010. 11.

19. De Vrese, M. and B. Offick, Probiotics and prebiotics: effects on diarrhea, in Bioactive Foods in Promoting Health. 2010, Elsevier. p. 205-227.

20. Guandalini, S., Probiotics for prevention and treatment of diarrhea. Journal of Clinical Gastroenterology, 2011. 45: p. S149-S153.

\section{Figures And Tables}

Fig. 1-Summary of patient flow diagram

Fig. 2-Comparison of diarrhea duration (days) from onset of treatment until recovery among the three statistical groups.

Due to technical limitations, tables 1-6 are only available as a download in the supplemental files section.

\section{Figures}




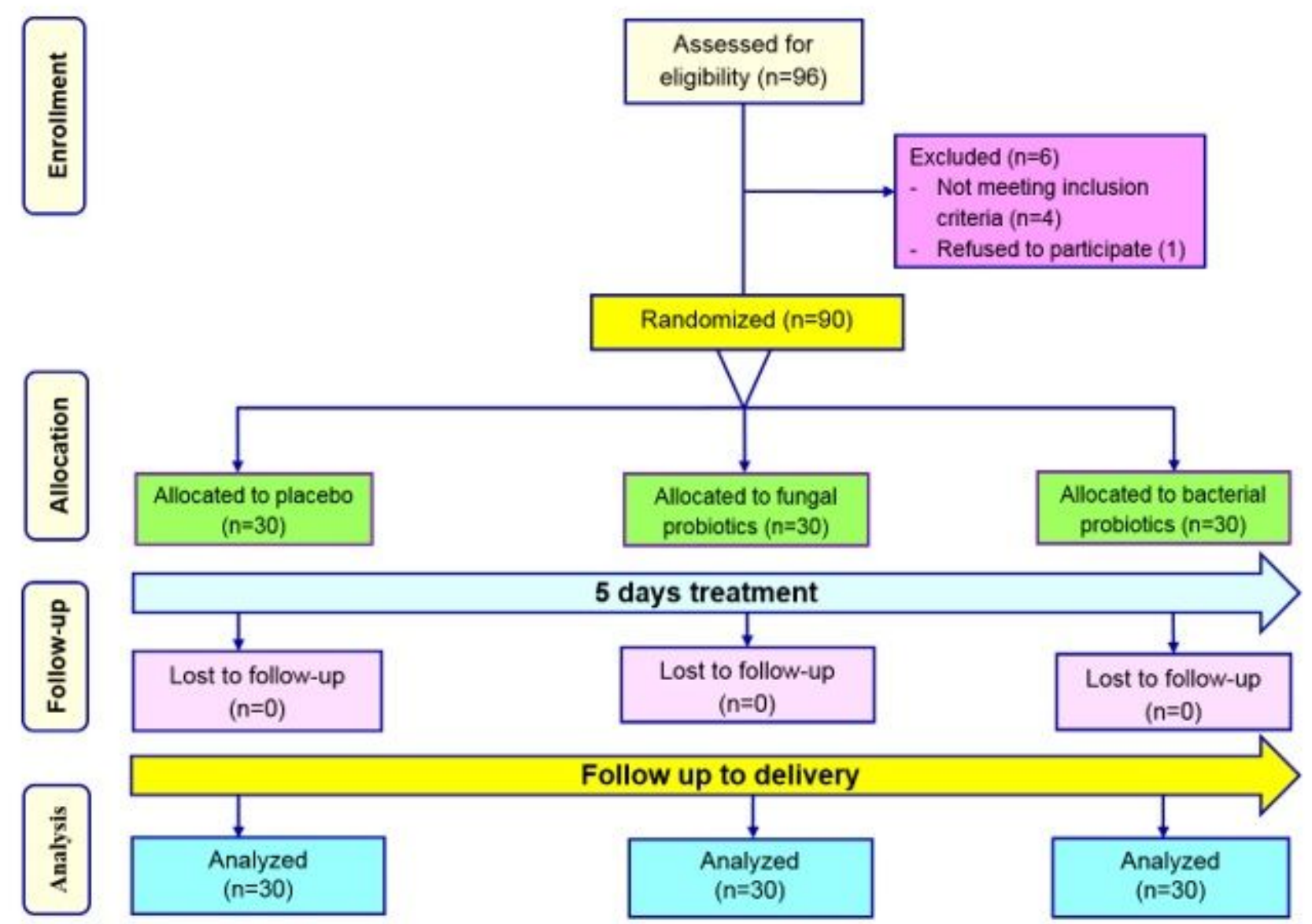

\section{Figure 1}

Summary of patient flow diagram 


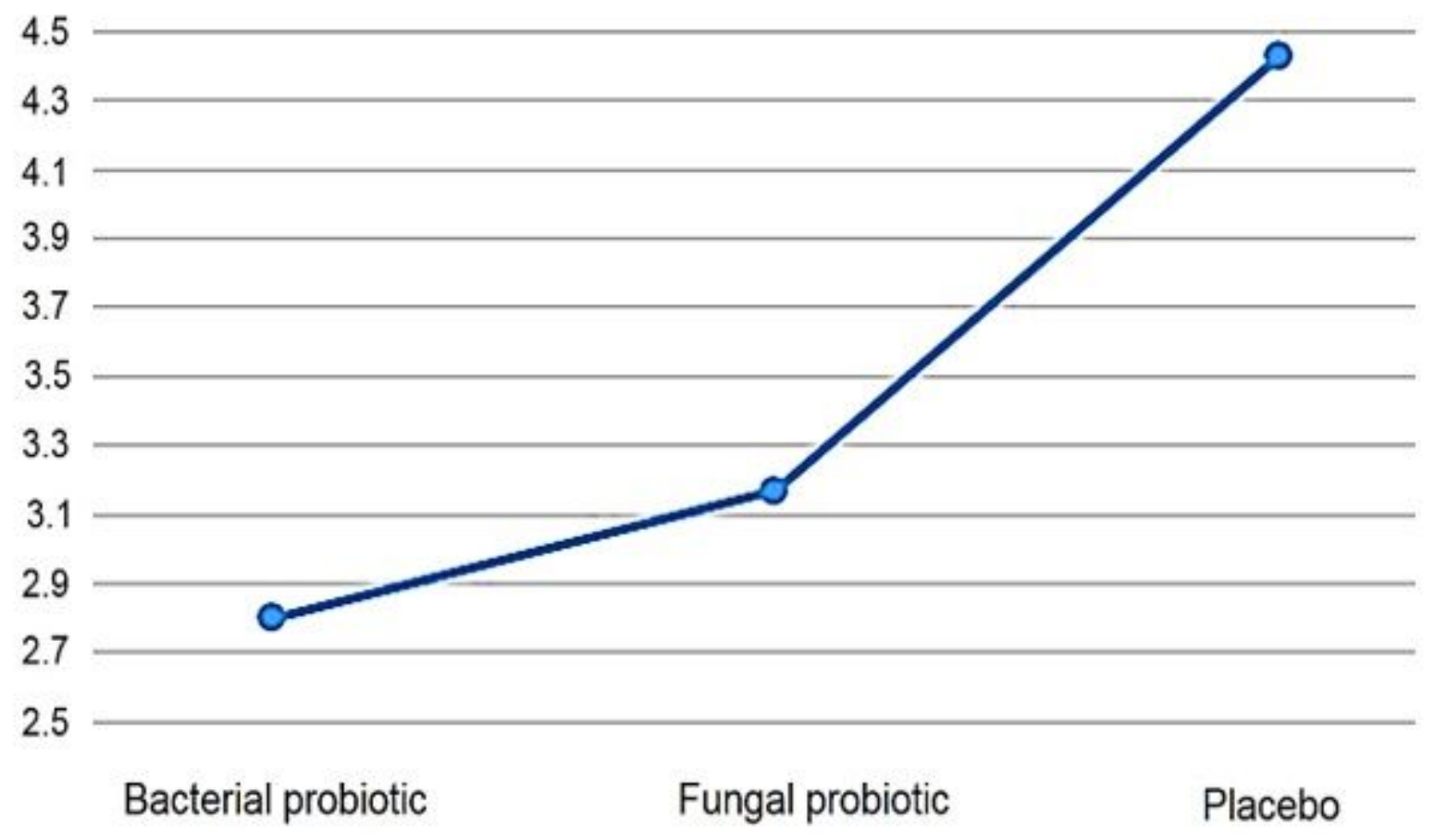

\section{Figure 2}

Comparison of diarrhea duration (days) from onset of treatment until recovery among the three statistical groups.

\section{Supplementary Files}

This is a list of supplementary files associated with this preprint. Click to download.

- Table6.jpg

- Table3.jpg

- Table4.jpg

- Table5.jpg

- Table2.jpg

- Table1.jpg 